\title{
Impiego della dialisi peritoneale nell'encefalopatia mitocondriale neurogastrointestinale (MNGIE): un caso clinico
}

\author{
M.P. Zito ${ }^{1}$, S. Maldone ${ }^{1}$, I. Capelli' ${ }^{2}$, F. Centofanti' ${ }^{2}$, C. Raimondi ${ }^{2}$
}

${ }^{1}$ Infermiere Day Service Peritoneale, Azienda Ospedaliero-Universitaria S. Orsola, Bologna

${ }^{2}$ Medico Unità Operativa di Nefrologia, Dialisi e Trapianto, Azienda Ospedaliero-Universitaria S. Orsola, Bologna, Direttore Prof. S. Stefoni

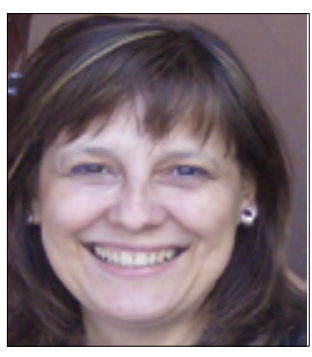

Maria Pia Zito

\section{Introduzione}

L'encefalomiopatia mitocondriale neurogastrointestinale (MNGIE) è una rara patologia ereditaria a trasmissione autosomica recessiva causata da mutazioni del gene ECGF1 situato sul braccio lungo del cromosoma 22 (1). Tale gene codifica per l'enzima citosolico Timidina-Fosforilasi (TP), il quale regola il catabolismo della timidina e della desossiuridina a timina e uracile. Le mutazioni note causano una perdita della funzione enzimatica ( $<10 \%$ dell'attività enzimatica media), con relativo aumento dei livelli sierici di timidina e desossiuridina e conseguente alterazione dei meccanismi di replicazione e riparazione del DNA mitocondriale, poiché la sintesi di quest'ultimo è maggiormente relata alla via di recupero della timidina-fosforilasi rispetto al DNA nucleare che predilige la sintesi de novo. La sindrome MNGIE è caratterizzata dall'associazione tra alterata motilità gastrointestinale, neuropatia periferica, oftalmoplegia esterna progressiva cronica e leucoencefalopatia. Sono stati descritti poco meno di 100 casi sporadici e familiari. I primi segni clinici di solito compaiono tra i 10 e i 40 anni (molto spesso prima dei 20 anni). I sintomi sono progressivi e il quadro clinico è contraddistinto da gravi disturbi gastrointestinali (crampi, vomito, diarrea, pseudo-ostruzione intestinale, disfagia e gastroparesi), secondari a difetti della motilità intestinale. I disturbi gastrointestinali evolvono gradual- mente verso la pseudo-ostruzione, che progredisce verso uno stato cachettico. Il coinvolgimento neurologico comprende l'oftalmoplegia progressiva cronica con o senza ptosi e la neuropatia sensitivo-motoria periferica. La sordità, la retinite pigmentosa e il coinvolgimento cerebellare sono meno frequenti e non sono evocativi della sindrome. La diagnosi si basa sul dosaggio dell'attività della timidina fosforilasi nei leucociti (assenza di attività nei soggetti sintomatici e riduzione di attività nei soggetti asintomatici eterozigoti) e sull'analisi genetica. L'iter diagnostico è caratterizzato dalla necessità del trattamento della pseudo-ostruzione intestinale cronica. La clearance della timidina e della deossiguanosina appare un approccio efficace. La terapia della MNGIE è ancora incerta e sia l'infusione d'immunoglobuline sia il trattamento corticosteroideo si sono dimostrati inefficaci (2). Il trattamento emodialitico, sperimentato nel tentativo di rimuovere i nucleosidi in eccesso, è gravato da un rebound precoce degli stessi metabolici subito dopo il trattamento (3). L'osservazione che l'infusione di piastrine può ridurre transitoriamente i livelli di nucleosidi, (4) ha portato all'effettuazione del trapianto del midollo allogenico come terapia risolutiva della sindrome (5). Tuttavia gli stessi autori riportano che un paziente sottoposto a trapianto è guarito mentre l'altro è deceduto per complicanze dovute al trapianto stesso. La prognosi è sfavorevole a causa della gravità del coinvolgimento digestivo, associato alle infezioni e alla necessità costante di ricorrere all'alimentazione parenterale. Riportiamo un caso di trattamento combinato con dialisi peritoneale (DP) e 


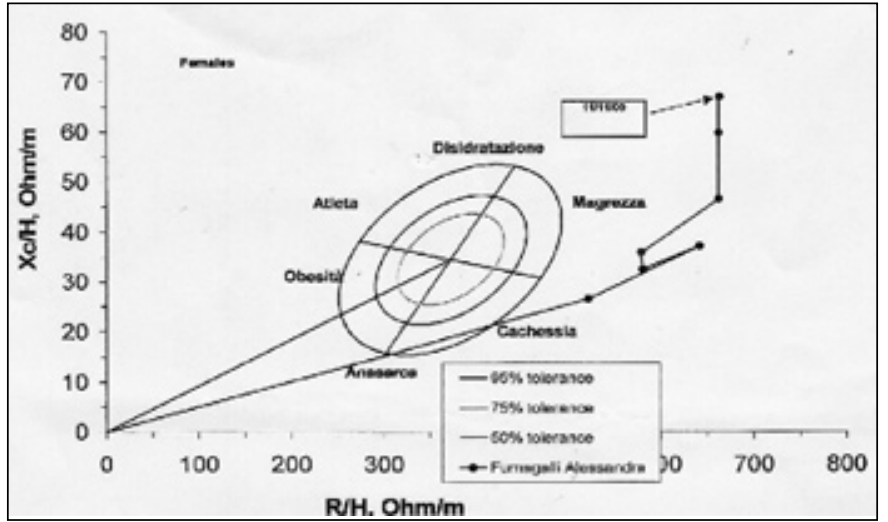

Fig. 1 - Il quadro impedenziometrico evidenzia una grave malnutrizione calorico-proteica e disidratazione.

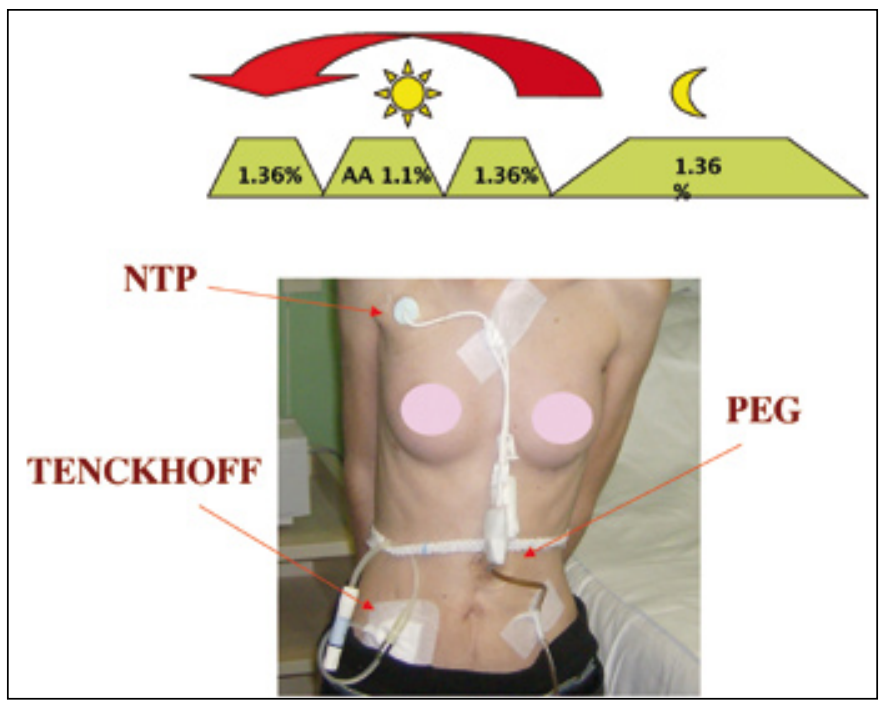

Fig. 2 - CAPD domiciliare a 4 scambi, volume di carico di $1000 \mathrm{ml}$. Uno dei quattro scambi viene effettuato con Nutrineal PD4 (AA 1.1\%).

nutrizione parenterale (NP) in una paziente affetta da MNGIE e severa malnutrizione.

\section{Caso Clinico}

Paziente di 27 anni che, sin dall'infanzia, presenta disturbi gastrointestinali e dolori addominali complicati da occlusioni intestinali recidivanti e severo quadro di malnutrizione (19 kg di peso corporeo). Proveniente da un'altra regione e dopo precedenti tentativi di porre una diagnosi etiologica e numerosi, infruttuosi tentativi terapeutici, si rivolge al Centro Regionale per l'insufficienza intestina-

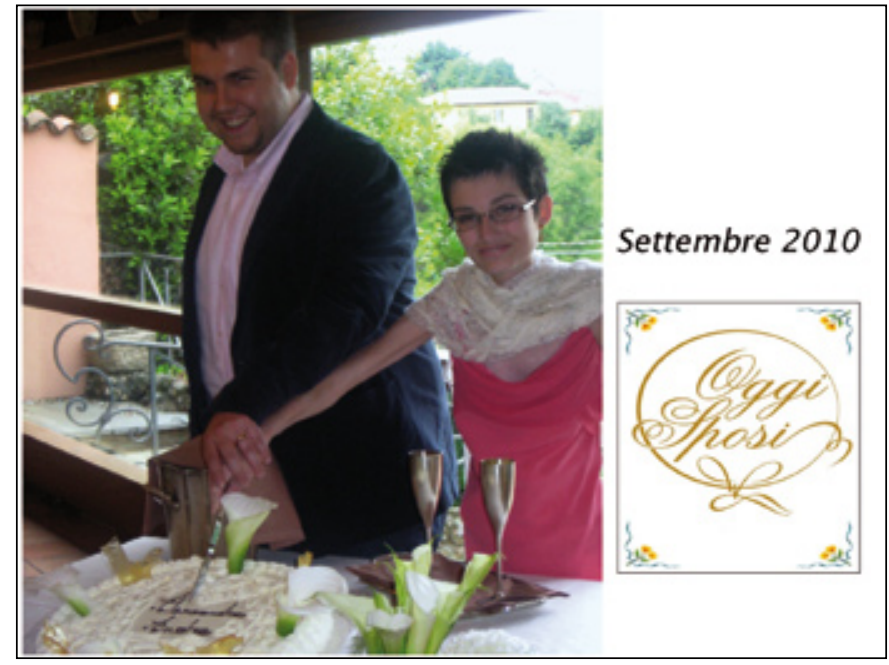

Fig. 3

le cronica del nostro Policlinico dove, visto il quadro di grave deperimento organico e di compromissione della deambulazione autonoma, viene effettuato un ricovero ospedaliero urgente. All'ingresso la paziente presenta un peso corporeo di $19 \mathrm{~kg}$, (BMI 8,8), protidemia totale $4,5 \mathrm{~g} / \mathrm{dl}$, albuminemia $2,0 \mathrm{~g} / \mathrm{dl}$, funzione renale nella norma (creatininemia $0.34 \mathrm{mg} / \mathrm{dl}$, Cockroft 78,5 ml/ $\mathrm{min})$. Il quadro impedenziometrico evidenzia una grave malnutrizione calorico-proteica e disidratazione (Fig. 1). L'esame TC dell'addome mostra una severa gastrectasia con ristagno liquido, anse del tenue mesenteriale ipotoniche e dilatate. Nel sospetto che tale quadro mostrasse elementi analoghi ad altri rari casi riscontrati in precedenza e riconosciuti di natura congenita, la paziente è sottoposta, tra gli altri accertamenti, a un test genetico, che permette di formulare diagnosi di MNGIE, quindi viene iniziata una nutrizione parenterale di lunga durata e posizionata una PEG decompressiva e, sulla base delle poche esperienze già riportate in letteratura, viene intrapreso un trattamento-ponte mediante DP in previsione di un trapianto di midollo osseo allogenico (Fig. 2).

Visto il soma ridotto della paziente, si posiziona, mediante laparoscopia, un catetere peritoneale tipo Tenchkoff di $14,5 \mathrm{~cm}$ nel tratto intraddominale e si imposta uno schema dialitico con carichi di volume ridotto: CAPD domiciliare a 4 scambi, volume di carico di $1000 \mathrm{ml}$. Uno dei quattro scambi viene effettuato con Nutrineal PD4 Baxter (AA 1,1\%). L'analisi del Test di Equilibrio Peritoneale (PET) dimostra una capacità di trasporto dei soluti della membrana peritoneale medio-alta.

Il campionamento di sangue, urine e liquido peritoneale mostrano dosaggi di timidina elevati:

- sangue 18,26 micromoli/L (normale $=0.21$ micromoli/L); 
- urine 187,23 micromoli/L corrispondente a una escrezione di $78.30 \mathrm{micromoli} / \mathrm{mmol}$ creatinina (escrezione normale $=1.65 \mathrm{micromoli} / \mathrm{mmol}$ creatinina);

- liquido peritoneale 4,38 micromoli/L (controllo su liquido peritoneale di paziente non affetto 0,05 micromoli/l).

A un anno di terapia la paziente presenta un rilevante miglioramento del quadro clinico, ripresa della deambulazione autonoma e parziale ripresa dell'alimentazione (da idrica a semisolida). Il peso corporeo ha raggiunto i 27,7 $\mathrm{kg}$ (BMI 12.1, protidemia totale 5,9 $\mathrm{g} / \mathrm{dl}$, albuminemia 2,8 $\mathrm{g} / \mathrm{dl}$ ) con miglioramento dei parametri antropometrici misurati mediante impedenziometria e aumento di massa grassa e di massa cellulare attiva con riduzione di acqua extracellulare $(\mathrm{R} / \mathrm{H}+26,5 \mathrm{ohm} / \mathrm{m})$. Non si sono verificate complicanze infettive legate al trattamento peritoneale e alla presenza contestuale della PEG e del CVC per la nutrizione parenterale. La rimozione di timidina peritoneale è risultata stabile nel corso del trattamento: dopo un anno timidina del liquido peritoneale di 2,81 micromoli/l.

A un anno dall'inizio dei trattamenti combinati, alla luce delle condizioni cliniche migliorate e in previsione del trapianto di midollo da eseguirsi entro poco tempo, è stato deciso di rimuovere il catetere peritoneale per eliminare un nuovo fattore di rischio infettivo.

\section{Discussione}

L'encefalomiopatia neurogastrointestinale mitocondriale (MNGIE) è una rara patologia ereditaria secondaria a perdita di funzione dell'enzima Timidina-Fosforilasi (TP), con relativo aumento dei livelli sierici di timidina e desossiuridina e conseguente alterazione dei meccanismi di replicazione e riparazione del DNA mitocondriale. La timidina, in condizioni fisiologiche, è filtrata dal glomerulo renale e riassorbita dal tubulo prossimale, con una clearance renale di circa il $20 \%$ rispetto alla clearance della creatinina. Nei soggetti sani si riscontrano basse concentrazioni di timidina urinaria, poiché i livelli circolanti di tale molecola sono nulli o molto bassi. Nei soggetti affetti da MNGIE le concentrazioni plasmatiche di timidina sono aumentate fino a 60 volte rispetto ai valori sierici dei soggetti non affetti e il riassorbimento tubulare della timidina contribuisce in parte alla patogenesi della MNGIE, aumentandone i livelli nel plasma. La timidina è un nucleoside formato da uno zucchero pentoso (D-ribosio o 2-desossi-D-ribosio) e dalla timina, una base azotata pirimidinica. Ha un peso molecolare di 242229 D. Tra le terapie proposte per tentare di ridurre la concentrazione ematica dei nucleotidi tossici, l'emodialisi non sembra essere una metodica efficiente, poiché dopo meno di 3 ore dal termine della seduta $\mathrm{i}$ livelli della timidina ritornano ai valori pre-trattamento. La dialisi peritoneale permette una rimozione di fluidi e tossine più graduale e costante ed è meglio tollerata dai pazienti. Nei rari casi riportati in letteratura, è dimostrata una buona clearance peritoneale della timidina, con concentrazioni ben quantificabili nel liquido peritoneale, confermata anche nel caso da noi riportato. Anche se Yavuz et al non hanno dimostrato una significativa riduzione dei livelli plasmatici di timidina e desossiuridina con il trattamento dialitico peritoneale (6), tutti i casi riportati, insieme alla nostra esperienza, confermano la possibilità che la dialisi peritoneale in associazione alla nutrizione parenterale, possa migliorare la clinica e le condizioni nutrizionali dei pazienti portatori di tale patologia (7). La scelta del tipo di soluzioni da utilizzare e delle modalità di scambio si è basata sul concetto di miglioramento della rimozione dei soluti nelle soste lunghe e del riassorbimento di glucosio e acqua dal liquido peritoneale, che, in questo caso, era auspicabile. La scelta della soluzione Nutrineal PD4 è stata fatta su base empirica e sulle esperienze effettuate nei pazienti con insufficienza renale cronica e MIA sindrome. Il volume di carico è stato individuato, dopo qualche tentativo, sulla base del peso corporeo e della tolleranza della paziente all'ingombro addominale che peggiorava i problemi di transito intestinale.

\section{Conclusioni}

Il trattamento combinato (NP/DP), nel caso descritto, ha consentito un miglioramento dello stato nutrizionale anche attraverso l'assorbimento di glucosio e aminoacidi dalla soluzione per dialisi peritoneale. Tale trattamento potrebbe, in casi analoghi, essere proposto come terapia-ponte in preparazione ad altre terapie, quali il trapianto di midollo. A livello infermieristico abbiamo modificato protocolli e procedure per adattarli al singolo caso. La paziente presentava una propria conoscenza per quello che riguardava le procedure per la medicazione della PEG e del CVC per l'alimentazione parenterale. Le procedure per la medicazione dell'exit-site e la metodica di dialisi peritoneale aumentavano l'impegno della paziente ma sono state analizzate insieme con lei, adattando i due metodi senza modifiche rilevanti. Questo ha contribuito al miglioramento della compliance al trattamento. Il gruppo infermieristico aveva poca conoscenza della malattia e si è adoperato per raccogliere informazioni scientifiche in merito per riportarle alla paziente che è sempre stata ottimista nei confronti del 
trattamento. Possiamo pertanto concludere che l'approccio dialitico peritoneale possa rappresentare una valida possibilità terapeutica temporanea per la MNGIE. Nel settembre 2010 la Paziente si è sposata (Fig. 3).

\section{Ringranziamenti}

Ringrazio la Paziente che ha permesso la pubblicazione delle sue foto, la collega Sabina Maldone, i Medici dell'U.O. che hanno collaborato nella stesura del lavoro e l'Associazione EDTNA/ERCA per aver accettato il lavoro come presentazione orale al Congresso Nazionale e come Poster alla Conferenza Europea EDTNA/ERCA di Ljubljana (Slovenia) 2011.

\section{Riassunto}

L'Encefalomiopatia Mitocondriale Neurogastrointestinale (MNGIE) è una rara malattia autosomica recessiva causata da mutazioni del gene ECGF1 che codifica per l'enzima Timidina-Fosforilasi, il quale regola il catabolismo della timidina e della desossiuridina. Tali mutazioni causano la perdita della funzione dell'enzima, conseguente aumento dei livelli plasmatici di timidina e deossiuridina e alterazione dei meccanismi di riparazione e replicazione del DNA mitocondriale. La MNGIE è caratterizzata da deficit neurologici (neuropatia periferica, leucoencefalomiopatia), oftalmoplegia bilaterale, ptosi palpebrale, dismotilità/atonia gastrointestinale con malassorbimento e malnutrizione. Riportiamo un caso di trattamento combinato con Dialisi Peritoneale (DP) e Nutrizione Parenterale (NP) in una paziente affetta da MNGIE e severa malnutrizione, con disturbi gastrointestinali e dolori addominali complicati da occlusioni intestinali recidivanti e severo quadro di malnutrizione. $\mathrm{E}$ stata quindi iniziata una NP e, un trattamento mediante DP. La scelta del tipo di soluzioni da utilizzare e delle modalità di scambio si è basata sul concetto di miglioramento della rimozione dei soluti nelle soste lunghe e del riassorbimento di glucosio e acqua dal liquido peritoneale, che, in questo caso, era auspicabile. La scelta della soluzione Nutrineal PD4 è stata fatta su base empirica e sulle esperienze effettuate nei pazienti con insufficienza renale cronica e MIA sindrome.

Parole chiave: Dialisi Peritoneale, Malattia Mitocondriale, Malattia Genetica, MNGIE Encefalomiopatia Mitocondriale
Indirizzo degli Autori:

Maria Pia Zito

Infermiere Case Manager, Day Service Peritoneale

Azienda Ospedaliero-Universitaria S. Orsola

Via Massarenti 9

40138 Bologna

mariapiazito@tin.it

\section{Bibliografia}

1. Hirano M, Yebenes J, Jones CA, et al. Mitochondrial neurogastrointestinal encephalomyopathy syndrome maps to chromosome 22q13.32-qter. Am J Hum Genet 1998, 63: 526-33.

2. Bedlack RS, Vu T, Hammans S, Sparr SA, Myers B, Morgenlander J, Hirano M. MNGIE neuropathy: five cases mimicking chronic inflammatory demyelinating polyneuropathy. Muscle Nerve 2004; 29(3): 364-8.

3. Spinazzola A, Marti R, Nishino I, Altered thymidine metabolism due to defects of thymidine phosphorylase. J Biol Chem 2002; 277: 4128-33.

4. Lara MC, Weiss B, Illa I, et al. Infusion of platelets transiently reduces nucleoside overload in MNGIE. Neurology 2006; 67: 1461-3; Epub 2006 Sep 13.

5. Hirano M, Martí R, Casali C, et al. Allogeneic stem cell transplantation corrects biochemical derangements in MNGIE. Neurology 2006 67: 1458-60; Epub 2006 Sep 13.

6. Yavuz H, Ozel A, Christensen M, et al. Treatment of mitochondrial neurogastrointestinal encephalomyopathy with dialysis. Arch Neurol 2007; 64: 435-8.

7. Guarena $\mathrm{C}$, Ariaudo C, Daidola G, et al. La dialisi peritoneale come approccio terapeutico alla MNGIE G Ital Nefrol 2009; 26(Suppl 47): S139-S145. 


\section{TEST di VERIFICA - 3}

\section{1) Cosa significa Encefalopatia Mitocondriale} Neuro-Gastrointestinale (MINGIE)?

a) L'encefalomiopatia mitocondriale neurogastrointestinale (MNGIE) è una rara patologia ereditaria a trasmissione autosomica recessiva causata da mutazioni del gene ECGF1 situato sul braccio lungo del cromosoma 22

b) L'encefalopatia mitocondriale neurogastrointestinale e una malattia genetica e colpisce solo l'apparato digerente

c) L'encefalopatia mitocondriale neurogastrointestinale e una malattia genetica e aggredisce solo il sistema neurovegetativo

d) Tutte le precedenti

\section{2) Come avviene la diagnosi di MNGIE?}

a) La diagnosi si basa sulle manifestazioni cliniche, sulla storia familiare e su test di laboratorio (misurazione dei livelli di timidina nel plasma e dosaggio dell'attività dell'enzima timidina fosforilasi nei globuli bianchi) e la ricerca molecolare di mutazione del gene ECGF 1

b) Può essere confermata solo per via molecolare

c) La diagnosi si basa solo sui sintomi come nausea, vomito e compromissione della deambulazione autonoma

d) Tutte le precedenti

3) Come si manifesta l'encefalopatia mitocondriale neurogastrointestinale?

a) L'encefalopatia mitocondriale neurogastrointestinale è una sindrome caratterizzata da una varietà di sintomi tra cui anomalie progressive della motilità intestinale (con episodi ricorrenti di diarrea e/o vomito)

b) L'encefalopatia mitocondriale neurogastrointestinale è una sindrome caratterizzata da deperimento dovuto a nausea, reflusso gastro-esofageo, senso di sazietà precoce e difficoltà a deglutire (disfagia)

c) L'encefalopatia mitocondriale neurogastrointestinale è una sindrome che determina oftalmoplegia (paralisi della muscolatura degli occhi) progressiva, neuropatia periferica con alterazione della sensibilità e debolezza delle estremità

d) Tutte le precedenti

Le risposte corrette alle domande sono a pag. 18 